\title{
Identification of radiogenic heat source distribution in the crust: A variational approach
}

\author{
$\mathrm{R}^{\mathrm{N}} \mathrm{SINGH}^{1}$ and AJAY MANGLIK ${ }^{2}$ \\ ${ }^{1}$ CSIR Centre for Mathematical Modelling and Computer Simulation, \\ Bangalore 560 037, India \\ Present address: National Environmental Engineering Research Institute, Nehru \\ Marg, Nagpur 440 020, India \\ ${ }^{2}$ National Geophysical Research Institute, Uppal Road, Hyderabad 500007, \\ India \\ email: rnsingh@cmmacs.ernet.in; dirneeri@ nagpur.dot.net.in; \\ postmast@csngri.ren.nic.in (Attn.: A Manglik)
}

\begin{abstract}
Radiogenic heat sources present in the continental crust contribute significantly to the total surface heat flow and temperature distribution in the crust. Various models for the depth distribution of radiogenic sources have been proposed. Among these models the exponential model has been shown to be an optimal, smooth model through the variational approach applied to the heat conduction equation. In the present work, a two-layered model of the crust is considered and heat transport by advection is included in the upper layer. The heat transport in the lower layer is by conduction only. Application of variational principle determines the nature of the radiogenic source distribution in both the layers. The results thus obtained indicate a radiogenic source distribution which is more complex than a simple exponential model.
\end{abstract}

Keywords. Radiogenic heat source distribution; variational approach; heat conduction equation; two-layered crustal model.

\section{Introduction}

Temperature plays an important role in shaping the lithospheric deformation by way of influencing the mechanical properties of rocks. In the continental crust, temperature distribution depends mainly on two factors; the heat supplied from the earth's interior, and the radiogenic heat sources present in the crust. The radiogenic sources contribute to about $40 \%$ of the surface heat flow in continental regions making this an important element in determining the crustal temperature distribution. Measurement of the concentration of these sources, however, is limited only to very shallow depths due to practical limitations. In the absence of direct measurements various models for the depth distribution of radiogenic heat sources distribution have been proposed in the literature (Birch et al 1968; Lachenbruch 1970; Lachenbruch \& Bunder 1971). Of these, the exponential and constant distribution models have gained more importance. These models also satisfy the empirical relationship 
between surface heat flow and surface radiogenic heat generation proposed by Birch et al (1968).

In an alternative approach, Singh \& Negi (1980) applied a variational approach to the heat conduction problem and showed that the exponential model is an optimal model that provides smooth variation of function with minimum rate of entropy production. Recently Bodri \& Cermak (1993) solved a similar heat flow problem by variational approach incorporating fluid circulation in the upper crust and concluded that the exponential model with an additional term is an optimal model for the radiogenic heat source distribution in this case also. They treated the crust as a single unit affected by fluid circulation. In the present work we consider a two-layered model of the continental crust. The fluid circulation takes place only in the top layer. The problem is solved by considering advection diffusion in the first layer and conduction in the second layer.

\section{Variational formulation}

In the variational approach of identifying the unknown parametric function of any physical system, the central concept is formulation of an integral over unknown functions and their derivatives which assumes a minimum value. This method originated first in solving problems of applied mechanics and has been used in the last two hundred years to solve many physical, engineering, biological, and sociological problems (Singh \& Negi 1980a). In the heat conduction problem (Singh \& Negi 1980a) a one-dimensional steady state energy transport equation was considered as:

$$
K \frac{\partial^{2} T}{\partial z^{2}}=-A(z)
$$

where $K, T, A$, and $z$ are thermal conductivity, temperature, radiogenic heat source distribution, and depth co-ordinate respectively. Equation (1) was converted into a first-order heat flow equation,

$$
\frac{\mathrm{d} Q}{\mathrm{~d} z}=-A(z)
$$

by applying the Fourier law $Q(z)=K . \mathrm{d} T / \mathrm{d} z$. By minimizing the function,

$$
F(A, Q, z)=\int_{0}^{\infty}\left(A^{2}+\alpha^{2} Q^{2}\right) \mathrm{d} z
$$

with the boundary conditions, $Q(z=0)=Q_{s}$ and $Q(z \rightarrow \infty)=Q^{*}$, an exponential form for the radiogenic sources distribution was obtained as:

$$
A(z)=A_{0} \exp (-\alpha z)
$$

where $A_{0}$ is the radiogenic source concentration at the surface $(z=0)$. The coefficient $\alpha$ is equal to $A_{0} /\left(Q_{s}-Q^{*}\right)$.

Bodri \& Cermak (1993) used porous media flow formulation to include fluid circulation in the crust and applied variational method to

$$
\nabla \cdot\left(k_{m} \nabla T\right)+\rho_{l} \mathbf{q} g+(1-\phi) A=\rho_{l} c_{l}(\mathbf{q} \cdot \nabla T),
$$

where

$$
\mathbf{q}=-\frac{\mathcal{K}}{\mu}\left(\nabla p-\rho_{l} g\right)
$$


and $k_{m}, \rho_{l}, c_{l}, \phi, \mathcal{K}, \mu$, and $p$ are thermal conductivity of the medium, density of fluid, specific heat of fluid, porosity, hydraulic permeability, viscosity, and pressure, respectively. By minimizing the function

$$
F(A, Q, \mathbf{q}, z)=\int_{0}^{H}\left[A^{2}+\alpha^{2}\left(Q^{2}+\beta \mathbf{q}^{2}\right)\right] \mathrm{d} z,
$$

where

$$
\beta=k_{m} T \mu / K,
$$

the radiogenic source distribution was obtained as:

$$
A(z)=A_{0} \exp (-z / D)+a,
$$

where $D=1 / a_{1}$ and

$$
\begin{aligned}
A_{0} & =\frac{1}{(1-\phi) D}\left(Q_{0}+a_{2} D^{2}\right)\left[1+\frac{\rho_{l} c_{l} \mathbf{q} D}{k_{m}}\right], \\
a & =\frac{\rho_{l} \mathbf{q} g}{(1-\phi)}\left[1-\left(\frac{\rho_{l} c_{l} \mathbf{q} D}{k_{m}}\right)^{2}\right], \\
a_{1}^{2} & =\left(\frac{\rho_{l} c_{l} \mathbf{q}}{k_{m}}\right)^{2}+\alpha^{2}(1-\phi)^{2}, \\
a_{2} & =\frac{\rho_{l}^{2} c_{l} \mathbf{q}^{2}}{k_{m}} g .
\end{aligned}
$$

In the above solution it is implicitly assumed that $H \rightarrow \infty$ and hence the term corresponding to $\exp (z / D)$ is ignored. For the present work the crust was divided into two domains to consider fluid circulation in the uppermost crust and the problem was solved as a two-layered one. A schematic diagram of the model is shown in figure 1.

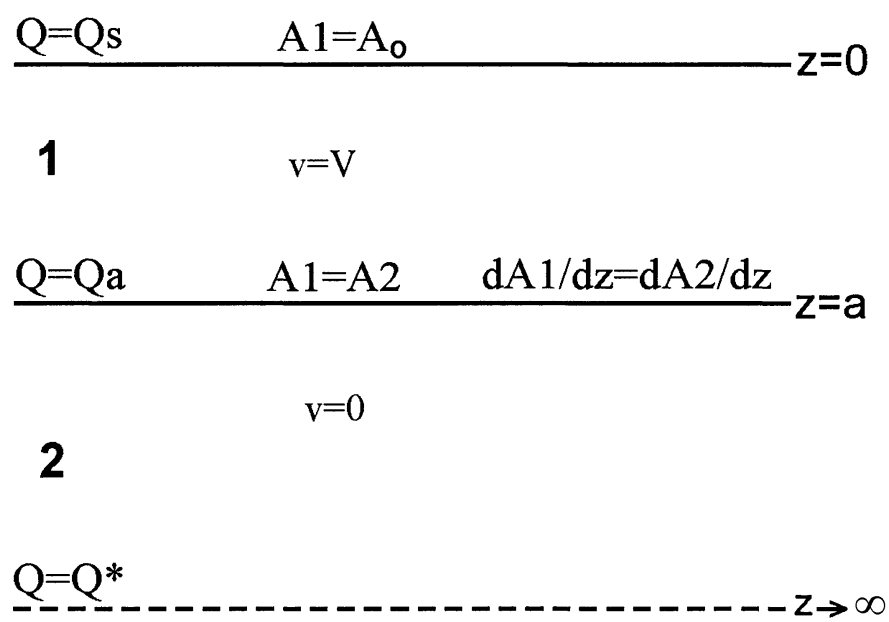

Figure 1. A schematic diagram of the model. 


\subsection{Domain-1}

In the first layer $(0 \leq z \leq a)$ the heat conduction equation in one-dimension with advective transport is given by

$$
K \cdot \frac{\partial^{2} T_{1}}{\partial z^{2}}+A_{1}(z)=\rho c V \frac{\partial T_{1}}{\partial z},
$$

where $T$ is temperature, $A(z)$ is the vertical distribution of radiogenic sources, $V$ is the advective velocity, and $z$ is the depth co-ordinate positive downward. The index 1 represents the first layer. Other parameters $K, \rho$, and $c$ are the thermal conductivity, density, and specific heat respectively. This second-order equation in temperature can be converted into a first-order equation in heat flux by using the following equation

$$
Q(z)=K \frac{\mathrm{d} T}{\mathrm{~d} z},
$$

where $Q(z)$ is the heat flux. In terms of heat flux, (8) can be expressed as

$$
\frac{\mathrm{d} Q_{1}}{\mathrm{~d} z}+\beta \cdot Q_{1}=-A_{1}(z),
$$

where $\beta=-\rho c V / K$. The boundary conditions are given as

$$
Q_{1}(z=0)=Q_{s} ; \quad Q_{1}(z=a)=Q_{a} .
$$

Following Singh \& Negi (1980a) and Bodri \& Cermak (1993) the following function is minimized

$$
F\left(A_{1}, \dot{A}_{1}, Q_{1}, \dot{Q}_{1}\right)=\int_{0}^{a}\left[\left(A_{1}^{2}+\alpha^{2} Q_{1}^{2}\right)+\lambda\left(\dot{Q}_{1}+\beta Q_{1}+A_{1}\right)\right] \mathrm{d} z
$$

where $\alpha$ and $\lambda$ are the constants to be determined and $\dot{Q}_{1}$ is $\mathrm{d} Q_{1} / \mathrm{d} z$. This gives smooth variation of $A_{1}(Z)$ within the layer and has minimum rate of entropy production.

The Euler form of (12) is obtained by applying the conditions (Burghes \& Graham 1980):

$$
\frac{\partial F}{\partial A_{1}}-\frac{\mathrm{d}}{\mathrm{d} z}\left(\frac{\partial F}{\partial \dot{A_{1}}}\right)=0
$$

and

$$
\frac{\partial F}{\partial Q_{1}}-\frac{\mathrm{d}}{\mathrm{d} z}\left(\frac{\partial F}{\partial \dot{Q}_{1}}\right)=0 .
$$

From (12 and 13)

$$
2 A_{1}+\lambda=0,
$$

and from (12 and 14) one gets:

$$
2 \alpha^{2} Q_{1}+\lambda \beta-\dot{\lambda}=0 .
$$

Eliminating $\lambda$ from (15) and (16) and substituting the expression for $A_{1}$ from (10) the resulting equation in terms of heat flux $Q_{1}$ becomes 


$$
\frac{\partial^{2} Q_{1}}{\partial z^{2}}-\left(\alpha^{2}+\beta^{2}\right) Q_{1}=0
$$

Solution of this equation can be written as

$$
Q_{1}(z)=C_{1} \exp (-\gamma z)+C_{2} \exp (\gamma z)
$$

where $\gamma=\left(\alpha^{2}+\beta^{2}\right)^{1 / 2}$ and $C_{1}$ and $C_{2}$ are the constants which can be evaluated by using the boundary conditions given by (11). The expressions for the heat flux and radiogenic sources distribution now become

$$
\begin{aligned}
& Q_{1}(z)=\frac{Q_{s}}{P}\left(e^{\gamma(a-z)}-e^{-\gamma(a-z)}\right)+\frac{Q_{a}}{P}\left(e^{\gamma z}-e^{-\gamma z}\right), \\
& A_{1}(z)=\frac{Q_{s}}{P}\left[(\gamma-\beta) e^{\gamma(a-z)}+(\gamma+\beta) e^{-\gamma(a-z)}\right]-\frac{Q_{a}}{P}\left[(\gamma+\beta) e^{\gamma z}+(\gamma-\beta) e^{-\gamma z}\right],
\end{aligned}
$$

where $P=\exp (\gamma a)-\exp (-\gamma a)$.

There are two unknowns $\gamma$ and $Q_{a}$ to be evaluated in order to describe the heat flux and radiogenic sources distribution in the layer.

\subsection{Domain-2}

In the second layer $(a \leq z \rightarrow \infty)$ the advection term is taken as zero i.e. $\beta=0$ and the first order heat flux equation can now be written as

$$
\frac{\mathrm{d} Q_{2}}{\mathrm{~d} z}=-A_{2}(z)
$$

along with the boundary conditions

$$
Q_{2}(z=a)=Q_{a} \quad \text { and } \quad Q_{2}(z \rightarrow \infty)=Q^{*} .
$$

In this case the function

$$
J\left(A_{2}, \dot{A}_{2}, Q_{2}, \dot{Q}_{2}\right)=\int_{a}^{\infty}\left[\left(A_{2}^{2}+\eta^{2} Q_{2}^{2}\right)+\lambda_{1}\left(\dot{Q}_{2}+A\right)\right] \mathrm{d} z,
$$

is minimized and, following the similar procedure as discussed above, the solution for heat flux and radiogenic sources is obtained as

$$
\begin{aligned}
& Q_{2}(z)=\left(Q_{a}-Q^{*}\right) e^{-\eta(z-a)}+Q^{*}, \\
& A_{2}(z)=\eta\left(Q_{a}-Q^{*}\right) e^{-\eta(z-a)} .
\end{aligned}
$$

These equations also have two unknowns $\eta$ and $Q_{a}$. The solution of the system, therefore, requires three conditions in order to evaluate $\gamma, \eta$, and $Q_{a}$.

Since the variational approach gives a smooth variation of the radiogenic sources with depth we assume the interface conditions

$$
A_{1}(z)=A_{2}(z) ; \quad \mathrm{d} A_{1} / \mathrm{d} z=\mathrm{d} A_{2} / \mathrm{d} z, \quad \text { at } \quad(z=a) .
$$



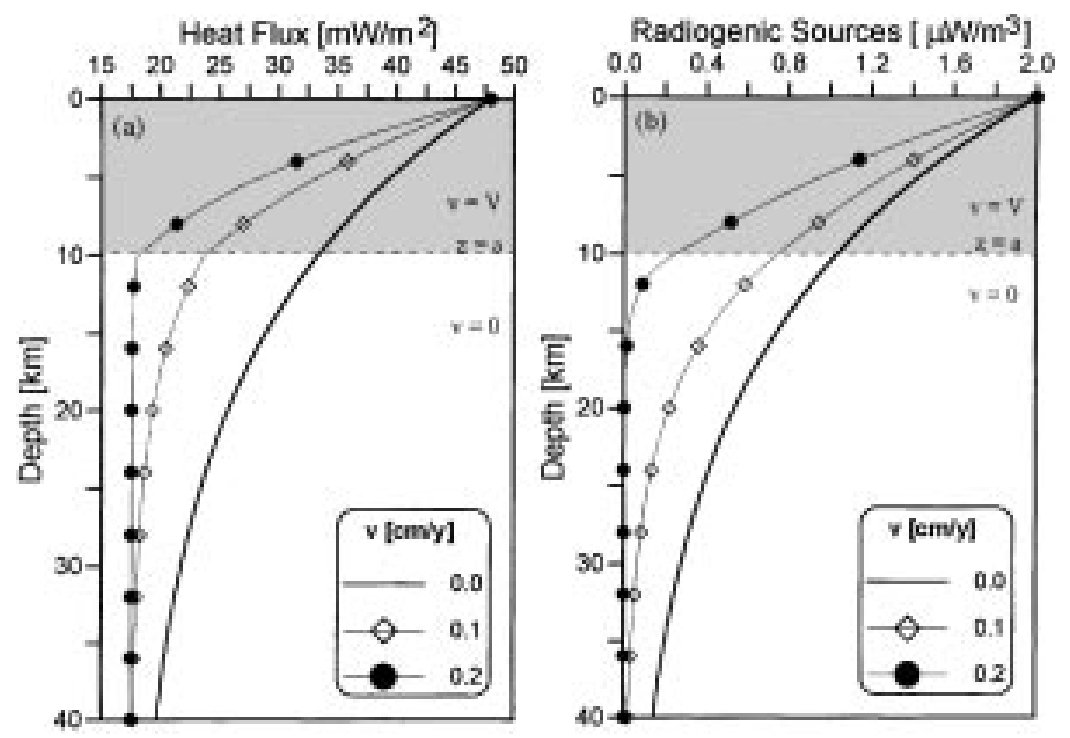

Figure 2. (a) Heat flux variation and (b) radiogenic heat source distribution with depth in the crust for different values of advective velocity $V$ in the upper crust.

The third equation is obtained by prescribing the surface value of radiogenic sources at $z=0$ as

$$
\frac{\gamma Q_{s}}{P}\left(e^{\gamma a}+e^{-\gamma a}\right)-\beta Q_{s}-\frac{2 \gamma Q_{a}}{P}=A_{0} .
$$

Elimination of $\eta$ and $Q_{a}$ from these three equations results in a nonlinear equation in terms of $\gamma$ as

$$
\left[2 \delta Q_{s}-(\delta \xi+\beta) R\right]^{2}+\left[2 \beta \delta Q_{s}-\left(\gamma^{2}+\delta \xi \beta\right) R\right]\left(R-Q^{*}\right)=0
$$

where $R=\left[(\xi \delta-\beta) Q_{s}-A_{0}\right] / 2 \delta, \xi=\exp (\gamma a)+\exp (-\gamma a)$, and $\delta=\gamma / P$. Once $\gamma$ is computed $\eta$ and $Q_{a}$ can also be obtained from (25).

\section{Results}

The results presented are for an example problem in which fluid circulation has been considered in the uppermost $10 \mathrm{~km}$ of the crust. The results were first computed for the zero velocity case $(\beta=0)$ using (19) and (20) and compared with those obtained by the analytical expression given by Singh \& Negi (1980b) and are shown in figure 2 . The parameters used in the computation are $\rho=3300 \mathrm{~kg} / \mathrm{m}^{3}, c=1.0 \mathrm{~kJ} / \mathrm{kg} . \mathrm{K}, K=3.0 \mathrm{~W} / \mathrm{m} . \mathrm{K}$, $Q_{s}=48 \mathrm{~mW} / \mathrm{m}^{2}, Q^{*}=17.5 \mathrm{~mW} / \mathrm{m}^{2}$, and $a=10 \mathrm{~km}$. The heat flux variation and radiogenic sources distribution are shown in figures $2 a$ and b, respectively. Since (27) is a nonlinear equation in $\gamma$ the roots of this equation were obtained by graphical method. The function gives two roots for this case as $\gamma=0.048,0.187$. Since the second root gives a negative value of $\eta$ which corresponds to unbounded solution only the first root was used to compute $\eta$ and $Q_{a}$. The values of these parameters are 0.070 and 32.88 respectively. These results support exponential model as an optimal model of radiogenic sources distribution. 


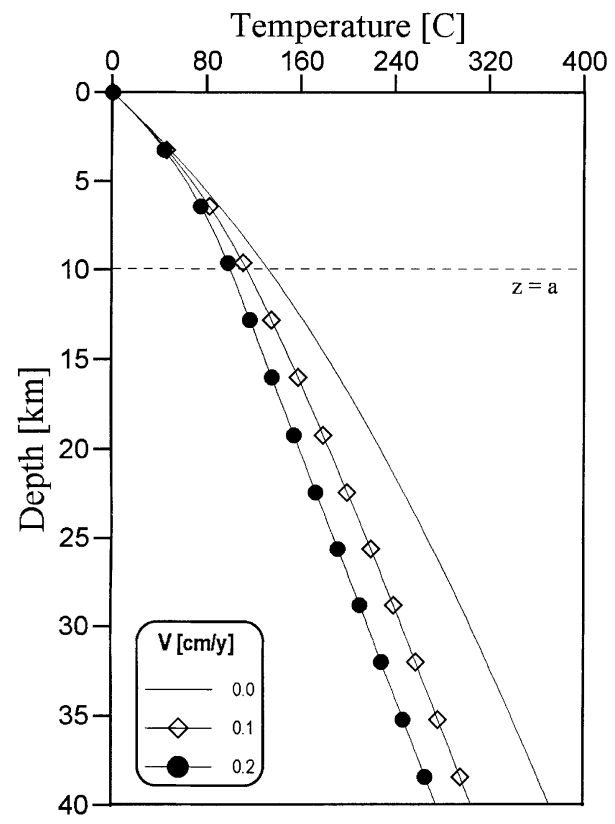

Figure 3. Temperature distribution with depth in the crust for different values of advective velocity $V$ in the upper crust.

Next, results were obtained for two values of advective velocity $V=0.1$ and $0.2 \mathrm{~cm} / \mathrm{y}$ ( $\beta=0.033$ and 0.066$)$. For $0.1 \mathrm{~cm} / \mathrm{y}$ velocity the values of $\gamma, \eta$, and $Q_{a}$ are $0.078,0.122$, and 23.64 respectively and for $0.2 \mathrm{~cm} / \mathrm{y}$ velocity these become $0.112,0.051$ and 18.0 respectively. These results are also shown in figure 2 . The results indicate that the radiogenic heat sources distribution is smooth in both the layers and follows exponential law in the second layer. In the first layer the distribution is more complicated. In case of large advective velocity, the concentration of these sources decreases drastically with depth. For example in the case of advective velocity of $0.2 \mathrm{~cm} / \mathrm{y}$ the radiogenic sources are confined mainly to the upper $10 \mathrm{~km}$ of the crust. The heat flux also decreases significantly with depth in case of advective flow in the upper crust.

The results of heat flow distribution shown in figure 2 were used to evaluate temperature variation with depth by integrating (9). These results, shown in figure 3 , indicate a reduction in temperature in presence of advection in the upper layer. The temperature at $40 \mathrm{~km}$ depth is reduced by $95^{\circ} \mathrm{C}$ in the case of $0.2 \mathrm{~cm} / \mathrm{y}$ advection velocity in comparison to that obtained by pure conduction. This result has implication for the continental lower crust because temperature at the Moho could be significantly reduced by introducing advective flow in the upper crust.

\section{Conclusion}

The variational approach applied to the one-dimensional thermal advection diffusion equation to analyse the nature of radiogenic sources distribution in the continental crust suggests that the distribution is not just a simple exponentially decreasing type in the upper crustal layer in presence of fluid flow. It is more complicated in nature and depends on the magnitude of advective velocity. In the lower crust where pure conduction mechanism is assumed 
the exponential law is an optimal model for radiogenic sources distribution. The results also indicate that the concentration of radiogenic sources at various depths can vary in presence of fluid advection even when the parameters surface heat flow $\left(Q_{s}\right)$, surface radiogenic source concentration $\left(A_{0}\right)$, and the reduced heat flow $\left(Q^{*}\right)$ remain constant. This has implications on the temperature distribution in the crust.

\section{References}

Birch F, Roy R F, Decker E R 1968 Heat flow and thermal history in New York and New England. In Studies of Appalachian geology: Northern and maritime (eds) E-an Zen, W S White, J B Hadley, J B Thompson Jr (New York: Interscience) pp 437-451

Bodri L, Cermak V 1993 Heat production in the continental lithosphere, part II: Variational approach. Tectonophysics 225: 29-34

Burghes D, Graham A 1980 Introduction to control theory including optimal control. Mathematics and its applications (New York: Ellis Horwood)

Lachenbruch A H 1970 Crustal temperature and heat production: Implication of the linear heat flow relation. J. Geophys. Res. 75: 3291-3300

Lachenbruch A H, Bunder C M 1971 Vertical gradient of heat production in the continental crust, some estimates from borehole data. J. Geophys. Res. 76: 3852-3860

Singh R N, Negi J G 1980a A variational approach to model the depth dependence of radiogenic heat in the crust. Geophys. Res. Lett. 7: 209-210

Singh R N, Negi J G 1980b A variational approach to model the depth dependence of radiogenic heat in the crust - addendum. Geophys. Res. Lett. 7: 559 